\title{
Cross-species transcriptional network analysis reveals conservation and variation in response to metal stress in cyanobacteria
}

Jiangxin Wang ${ }^{1,2}$, Gang Wu ${ }^{3}$, Lei Chen ${ }^{1,2}$ and Weiwen Zhang ${ }^{1,2^{*}}$

\begin{abstract}
Background: As one of the most dominant bacterial groups on Earth, cyanobacteria play a pivotal role in the global carbon cycling and the Earth atmosphere composition. Understanding their molecular responses to environmental perturbations has important scientific and environmental values. Since important biological processes or networks are often evolutionarily conserved, the cross-species transcriptional network analysis offers a useful strategy to decipher conserved and species-specific transcriptional mechanisms that cells utilize to deal with various biotic and abiotic disturbances, and it will eventually lead to a better understanding of associated adaptation and regulatory networks.

Results: In this study, the Weighted Gene Co-expression Network Analysis (WGCNA) approach was used to establish transcriptional networks for four important cyanobacteria species under metal stress, including iron depletion and high copper conditions. Cross-species network comparison led to discovery of several core response modules and genes possibly essential to metal stress, as well as species-specific hub genes for metal stresses in different cyanobacteria species, shedding light on survival strategies of cyanobacteria responding to different environmental perturbations.

Conclusions: The WGCNA analysis demonstrated that the application of cross-species transcriptional network analysis will lead to novel insights to molecular response to environmental changes which will otherwise not be achieved by analyzing data from a single species.
\end{abstract}

Keywords: Cross-species, Transcriptional network, Metal stress, Cyanobacteria

\section{Background}

Many biological systems operate in a similar manner across a large number of species or conditions [1]. Cross-species analysis of genomic sequences has made fundamental contributions to modern biology in defining the putative function of new genes [2]. Recent exponential growth in microarray expression datasets allows researchers to combine expression experiments from multiple species to identify genes that are not only conserved in sequence but also operated in a similar way [3]. In contrast to the static sequence measurements,

\footnotetext{
* Correspondence: wwzhang8@tju.edu.cn

'School of Chemical Engineering \& Technology, Tianjin University, Tianjin 300072, People's Republic of China

${ }^{2}$ Key Laboratory of Systems Bioengineering, Ministry of Education, Tianjin 300072, People's Republic of China

Full list of author information is available at the end of the article
}

microarrays measure the dynamic, condition-specific responses of complex biological systems [4-7], and their comparative analysis has led to improvements in annotating gene function and inferring the evolution of interaction and regulatory networks [3]. In addition, crossspecies microarray analysis also allowed identification of core transcriptional networks, and their conservation and variation in closely related species $[8,9]$.

Correlation networks are increasingly being used in various bioinformatics applications [10-12]. Among them, Weighted Gene Co-expression Network Analysis (WGCNA) is a computational method to describe the correlation patterns among genes across transcriptomic datasets $[11,13]$. WGCNA has been used in identifying functional clusters (modules) of highly correlated genes, summarizing such clusters using the module eigengene or an intramodular hub gene, relating modules to one

\section{Biomed Central}


another and to external sample traits (using eigengene network methodology), calculating module membership measures in many systems, and correlation network analysis can also be used to determine biological correlated candidate biomarkers or disease therapeutic targets [11]. The WGCNA approach has been applied in many eukaryotic systems, successfully linking molecular targets to oncogenic signals [14], complex traits [15], analyzing network divergence between human and chimpanzee neural patterns [16], and even comparing crossspecies gene expression in animals recently [17]. A recent comparative study of different network analysis methods indicated that WGCNA could be used not only for constructing gene networks, but also for detecting modules/sub-networks, identifying hub genes and selecting candidate genes as biomarkers, using Escherichia coli as an empirical sample [18].

As important primary producers and significant contributors of fixed carbon budget in many terrestrial and marine environments, cyanobacteria have been present in many different environments from coast to open sea for $\sim 2.5$ billion years [19]. Currently the marine cyanobacteria, Synechococcus and Prochlorococcus species are together responsible for at least $20 \%$ of global carbon fixation [20]. They play such significant roles in the carbon cycle that it is essential to understand to what environmental stresses they are susceptible and how they respond. Metal is one important environmental factor for cyanobacteria. For instance, iron is required for photosynthesis, and its limitation and restriction of primary productivity have been reported and has been considered as one important factor in the ecology of cyanobacteria [21,22]. Early studies have found the limitation of Prochlorococcus cell division rates by iron in the equatorial Pacific sea [23], and variations in the abundance of Prochlorococcus iron-related genes between oceans [24]. A recent study on transcriptomic response of high- and low-light-adapted Prochlorococcus strains to changing iron availability suggested high divergence of gene expression within strains [25]. The physiology and biochemistry associated with the iron-limited continuous culture of the halotolerant cyanobacterium Synechococcus PCC 7002 were also examined [26], and the regulatory network for acclimation of the obligate photoautotrophic fresh water cyanobacterium $S$. elongatus PCC 7942 to iron limitation was studied by transcript profiling recently [27]. Interestingly, oceanic Synechococcus strains were found much more sensitive to iron limitation than coastal strains [28]. Copper is another metal commonly present in natural environments. It has been reported that different cyanobacteria species have varying levels of copper tolerance, with Prochlorococcus species considered to be more sensitive to copper than Synechococcus species [29]. Within
Synechococcus species, copper tolerance has also varied significantly [30], with coastal species of Synechococcus species exhibiting increased tolerance to copper shock and a distinctive transcriptional responses relative to those of open-ocean species [31].

To seek better understanding of molecular response of cyanobacteria to metal stress, as a proof of concept we applied a cross-species transcriptional network analysis to four important cyanobacteria species under two different conditions of metal stress, Prochlorococcus MED4 (PMM) and Prochlorococcus MIT9313 (PMT) under stress of iron depletion [25], Synechococcus 9311 (SYG) and Synechococcus WH 8102 (SYW) under stress of high copper concentration [31]. Transcriptional networks for each individual species were constructed using a WGCNA method [11,13], and then cross-strain analysis was performed to reveal the conservation and variation in terms of the response to iron and copper stresses among all species. In this study, cross-species analyses of two Synechococcus species under high copper stress condition, and two Prochlorococcus species under iron depletion stress condition were first performed separately, the results revealed the signature responses to iron in Synechococcus and to copper in Prochlorococcus, respectively. Then a cross-species comparison was conducted for all four cyanobacteria species under two different types of metal stresses. Notably, the results showed that 9 genes were commonly regulated in all four species used in this study. Although still needs more experimental evidences, the set of genes may represent an important core signature response to metals in these four cyanobacterial species. Interestingly, the species-specific hub genes detected in four species showed no overlap between each other, indicating possible species-specific strategy for metal acclimation in each species. Thus, the analysis demonstrated that the application of crossspecies transcriptional network analysis could lead to novel insights into molecular response to environmental changes which will otherwise not be achieved by analyzing data from a single species.

\section{Results and discussion}

\section{Construction of cyanobacteria metal response networks}

Comparison of transcriptional networks between different cyanobacteria species could provide valuable insights into the core and important responses to environmental stress at the molecular levels. To this end, we first sought to compile a complete transcriptional profiling datasets of different cyanobacteria species under iron depletion and copper toxicity. The datasets used in our analysis included transcriptional measurements of a total of 50 samples from 4 cyanobacteria species (i.e. PMM, PMT, SYG, SYW), representing iron depletion (PMT and PMM) and high copper toxicity (SYG and SYW) 
treatments, respectively (Table 1 ) [25,31]. To ensure that our dataset were reasonably selected, we also compared the treatment sample datasets with their controls through a clustering analysis. The results showed that all stress-treated datasets tended to be grouped together and can be visibly separated from the controls, suggesting the stresses of iron depletion and copper toxicity have caused significant changes at the transcriptional level (Additional file 1: Figure S1). Next, we applied the datasets of each species for the transcriptional network construction using a WGCNA method. Analysis of the transcriptional networks showed that a total of 17, 21, 32 and 28 distinct transcriptional modules can be detected within the transcriptional networks of PMT, PMM, SYG and SYW, respectively (Additional file 2: Figure S2). More transcriptional modules detected in SYG and SYW than PMT and PMM were probably due to the relatively large genome sizes of Synechococcus [32-34]. The association analysis between the phenotypes (i.e. iron or copper stress) and the detected modules showed that the distinguished transcriptional modules highly associated with the phenotypes can be identified in each of the four species according to the correlation coefficients ( $r$ value) and their confidence (low $p$-values) (Additional file 3: Figure S3). Among all transcriptional modules detected in each species, we found that 5 of 17 and 6 of 21 detected modules significantly correlated with iron depletion condition in PMT and PMM, respectively (Table 2); and 12 of 32 and 11 of 28 detected modules significantly correlated with the high copper toxicity in SYG and SYW, respectively (Table 2). The KEGG pathway analysis of these phenotype-correlated modules in different species showed that a total of 62-79 KEGG pathways were involved in these modules (Table 2). Interestingly, 69 and 49 KEGG shared pathways were detected in PMT/PMM to iron depletion and SYG/SYW to high copper toxicity, respectively (Table 2).

Several cyanobacteria species produce secondary metabolites such as xanthophyll carotenoids and their synthetic genes are often up-regulated in response to high light intensity, ultraviolet radiation, and desiccation [35]. The ability to exist in two redox states makes iron an essential cofactor for proteins involved in numerous major cellular processes such as respiration, amino acid metabolism and DNA metabolism. For instance, iron starvation down-regulated most amino acid biosynthesis related genes since many enzymes are iron dependent $[36,37]$. It was also reported that iron starvation also induced DNA recombination and DNA repair process in a Gram-negative diplococci bacteria [38]. PMT was originally isolated from the gulfstream 135 meter in depth (low light adapted) and could grow with iron of an order of magnitude lower [25]. Analysis of the iron-responsive modules suggested that the significant down-regulation of "amino acid metabolism" and "microbial metabolism in diverse environments" probably reduced the iron requirement, and the up-regulation of the "metabolism of secondary metabolites" protected the cells from oxidative stress caused by iron depletion directly or indirectly. At the same time, the up-regulation of "homologous recombination" might result in an increase of DNA mismatch repair and recombination [39]. Detailed analysis of genes with the same change trends (up- or downregulated) in both PMM and PMT under iron depletion condition suggested the mechanism that cyanobacteria employ to iron deficiency could be: firstly transporters were activated to obtain more iron from the environment [25], and photosynthesis and chlorophyll metabolism was inhibited due to the limited supply of iron [40-42], and then oxidative stress was enhanced and may cause RNA degradation, protein translation inhibition and other cellular metabolic changes. All these further resulted in inhibition of DNA replication and cell cycle block, and eventually growth cease.

The copper-responsive SYW yellow module showed a significantly high correlation between "ribosome biosynthesis and assembly" (syw03010, total 20 related genes enriched) and the copper toxicity treatments ( $p=6.78 \mathrm{E}$ $15)$, suggesting an involvement of protein synthesis program in copper acclimation (Additional file 4: Table S1). Another copper-responsive SYW greenyellow module showed a significant correlation with the "aminoacyltRNA biosynthesis" and "citrate cycle" (TCA cycle) pathways, which was characterized as a down-regulation response. According to KEGG pathway analysis, genes in this module tended to encode proteins involved in amino acid metabolism ( $p=0.0012 \sim 0.0372$ ) (Additional file 5: Table S2). The genes involved in "polycyclic aromatic hydrocarbon degradation" were identified in the copper-responsive SYG darkgreen module. The module also showed a significant correlation with amino acid

Table 1 Cyanobacteria species and related datasets used in this study

\begin{tabular}{lcccc}
\hline \multicolumn{1}{c}{ Species } & Code & Condition (GEO ID) & Original sample size & Included in this study after filtering \\
\hline Synechococcus 9311 & SYG & Copper toxicity (GSE13910) & 14 & 14 \\
Synechococcus WH 8102 & SYW & Copper toxicity (GSE13910) & 10 & 10 \\
Prochlorococcus MED4 & PMM & Iron depletion (GSE26533) & 16 & 10 \\
Prochlorococcus MIT 9313 & PMT & Iron depletion (GSE26533) & 23 & 16 \\
\hline
\end{tabular}




\begin{tabular}{|c|c|c|c|c|}
\hline $\begin{array}{l}\text { Metal } \\
\text { stress }\end{array}$ & Species & $\begin{array}{l}\text { Modules related to } \\
\text { phenotype }\end{array}$ & $\begin{array}{c}\text { KEGG } \\
\text { Pathways }\end{array}$ & $\begin{array}{l}\text { Common } \\
\text { pathways }\end{array}$ \\
\hline \multirow[t]{2}{*}{ Iron } & PMT & 5 & 79 & 65 \\
\hline & PMM & 6 & 68 & \\
\hline \multirow[t]{2}{*}{ Copper } & SYW & 12 & 63 & 49 \\
\hline & SYG & 11 & 62 & \\
\hline
\end{tabular}

metabolism (histidine, tyrosine, cysteine and methionine, arginine and proline; $p=5.59 \mathrm{E}-05 \sim 0.005)$. KEGG pathway analysis indicated that the SYG darkgreen module was enriched with genes encoding photosynthesis antenna proteins, and genes related to "microbial metabolism in diverse environments" and "oxidative phosphorylation" (Additional file 6: Table S3). The copper-responsive SYG brown module showed significant correlation with the "curated two-component", overrepresented with genes participating in signal transduction activity, including "response regulator", "two component system", "histidine kinase" and "ABC transporters". (Additional file 7: Table S4), suggesting the strong involvement of signal transduction systems in copper response in SYG species.

For high copper toxicity, a recent study showed that costal strains of marine Synechococcus species (SYG in this study) exhibited increased tolerance to copper shock and a distinctive transcriptional response relative to those of open-ocean strains (SYW) [31]. SYG was predicated to be more metal-tolerant and may be better in adapting to fast changing environments, including biotic and abiotic environmental stresses besides copper. Early studies showed that toxicity of excess copper may be arose through several mechanisms: production of reactive oxygen species (ROS), metal competitive binding and photosynthesis inhibition $[31,43,44]$. In this study, we found that significant copper-responsive modules existed in both species of SYG and SYW. Modules of SYW greenyellow and SYG darkgreen both contained genes related to "amino acid metabolism" and "microbial metabolism in diverse environments", representing a general stress response. Meanwhile, more specific responses were also found in SYW yellow module which contained a down-regulated cluster of ribosomal genes, and in SYG brown module which had up-regulated signal transduction and response, such as "curated twocomponent system", RR and HK system. The overlap of orthologous genes between SYW and SYG was modest, with only 46 orthologous genes being significantly regulated by high copper. Based on these results, we proposed that a possible strategy for SYW cells to survive under high copper condition was to slow down de novo protein synthesis and employ oxidative protection to eliminate the ROS damage caused by copper treatment, while SYW cells may use relatively active signal transduction and response systems to adjust its metabolism to adapt the changing environments.

\section{Signature genes and pathways of different cyanobacteria} species under metal stresses

Using WGCNA, we obtained modules that contained an exact number of assigned genes. The same genes could be assigned to multiple modules, although the association strengths in different modules could vary. Our results showed a high degree of cross-species module similarity between PMM and PMT under iron depletion condition, and between SYW and SYG under high copper condition, suggesting possible core responses to each treatment. In addition, the overlaps between iron depletion and copper toxicity response modules were also observed, suggesting the possible core and general response to metals and possible cross-talking response networks between these four species to different metal stresses. We identified the shared genes between response modules in different cyanobacteria species based on the significance of module membership values (Additional file 8: Figure S4, Additional file 9: Table S5 and Additional file 10: Table S6). The results showed that a total of 440 and 430 genes were found correlated with iron depletion in PMM and PMT, respectively, with only 34 of them shared between PMM and PMT with confident low $p$-values (Additional file 8: Figure S4A). Similarly, the numbers of the copper correlated genes were 736 and 580 in SYG and SYW, respectively (Additional file 8: Figure S4B), with only 70 of them shared between SYG and SYW.

In a recent study, only 4 of 1159 orthologous genes of PMT and PMM were found differentially regulated in response to iron in both species (using fold change > 2.0), although expression level of over a hundred genes changed in two species, suggesting a great diversity in terms of iron adaptation among different Prochlorococcus species [25]. Interestingly, only 1 pair (petF, ferredoxin, PMM0898/PMT1429) of 4 differentially expressed genes (petF, isiB PMM1171/PMT0801, hli05/08 PMM1404/ PMT1154, and idiA PMM1164/PMT0287) in iron stress previously reported [25] was showed in our shared gene list, due to the low correlation coefficient of the other three genes in the modules with $r$ ranging from 0.001 to 0.42 . Conventionally for microarray analysis, a cutoff of $>2$-fold change was commonly used; it is quite possible that some of the genes with important functions will have a smaller change (fold change < 2.0) and thus will be excluded. In this study, we focused on the coexpression property across two different species, thus no fold change threshold was applied, by doing this we could avoid information loss due to artificial fold- 
change cutoff (i.e. genes changed with significantly low $p$ values, but with less than 2.0 fold changes). Instead, we focused on only those genes in highly correlated modules to explore their possible functions in metal acclimation.

Interestingly, our cross-species analysis revealed 9 commonly shared genes among these four cyanobacteria species under two different metal stress conditions (Table 3). They were ABC transporter ATP binding component, carboxypeptidase Taq metallopeptidase, cell division protein $(f t s W)$, ferredixin, isocitrate dehydrogenase $\left(\mathrm{NAD}^{+}\right)(i d h 3)$, LysM domain, phosphoribosylaminoimidazolecarboxamide formyltransferase/IMP cyclohydrolase ( $p u r H)$, phycocyanob ilin:ferredoxin oxidoreductase $(p c y A)$ and 6-pyruvoyl

Table 3 Genes responsive in all four cyanobacteria species

\begin{tabular}{|c|c|c|c|c|c|c|c|}
\hline KO_ID & Name & Taxon & Gene & Module & $\begin{array}{l}\text { Module } \\
\text { cor }\end{array}$ & $\begin{array}{c}\text { Fold change } \\
(\log 2)\end{array}$ & p-value \\
\hline \multirow[t]{4}{*}{ K01737 } & \multirow[t]{4}{*}{ 6-pyruvoyl tetrahydrobiopterin synthase } & PMM & PMM0106 & turquoise & -0.755 & -0.5 & 0.024 \\
\hline & & PMT & РMT0164 & turquoise & -0.611 & 0.3 & 0.0009 \\
\hline & & SYW & SYNW1504 & black & 0.791 & 1.5 & 0.013 \\
\hline & & SYG & sync_1898 & brown & 0.977 & 0.2 & 0.002 \\
\hline \multirow[t]{4}{*}{ K00602 } & \multirow{4}{*}{$\begin{array}{l}\text { phosphoribosylaminoimidazolecarboxamide formyltransferase / } \\
\text { IMP cyclohydrolase }\end{array}$} & PMM & PMM0266 & magenta & 0.714 & 0.3 & 0.028 \\
\hline & & PMT & PMT1857 & purple & 0.613 & 0.3 & 0.025 \\
\hline & & SYG & sync_0289 & turquoise & -0.806 & -0.4 & 0.0001 \\
\hline & & SYW & SYNW0249 & greenyellow & -0.957 & -0.6 & 0.029 \\
\hline \multirow[t]{4}{*}{ K00030 } & \multirow[t]{4}{*}{ isocitrate dehydrogenase (NAD+) } & PMM & PMM1596 & magenta & 0.714 & 0.4 & 0.008 \\
\hline & & PMT & PMT1935 & turquoise & -0.611 & -0.2 & 0.006 \\
\hline & & SYG & sync_0214 & pink & 0.757 & -0.5 & 0.002 \\
\hline & & SYW & SYNW0166 & yellow & -0.964 & -0.5 & 0.001 \\
\hline \multirow[t]{4}{*}{ K03588 } & \multirow[t]{4}{*}{ cell division protein FtsW } & PMM & PMM1458 & green & 0.661 & -0.6 & 0.023 \\
\hline & & PMT & PMT1475 & turquoise & -0.611 & -0.2 & 0.027 \\
\hline & & SYG & sync_2326 & turquoise & -0.806 & -0.3 & 0.012 \\
\hline & & SYW & SYNW0475 & yellow & -0.964 & -0.2 & 0.011 \\
\hline \multirow[t]{4}{*}{ K05371 } & \multirow[t]{4}{*}{ phycocyanobilin:ferredoxin oxidoreductase } & PMM & PMM0747 & cyan & 0.743 & -0.3 & 0.0003 \\
\hline & & PMT & РMT0590 & pink & 0.695 & 0.3 & 0.026 \\
\hline & & SYG & sync_1656 & turquoise & -0.806 & -0.4 & 0.0011 \\
\hline & & SYW & SYNW1084 & brown & 0.869 & 0.2 & 0.048618 \\
\hline \multirow[t]{4}{*}{ K01299 } & \multirow[t]{4}{*}{ carboxypeptidase Taq (M32) metallopeptidase } & PMM & PMM0493 & red & -0.813 & -0.8 & 0.015 \\
\hline & & PMT & PMT1279 & greenyellow & -0.955 & -0.3 & 0.0005 \\
\hline & & SYG & sync_2040 & purple & -0.808 & -0.4 & 0.0008 \\
\hline & & SYW & SYNW1098 & greenyellow & -0.957 & 0.3 & $6.78 \mathrm{E}-05$ \\
\hline \multirow[t]{4}{*}{ - } & \multirow[t]{4}{*}{ LysM domain } & PMM & PMM0330 & turquoise & -0.755 & 0.7 & 0.003 \\
\hline & & PMT & РМТ0190 & turquoise & -0.611 & -0.2 & 0.004 \\
\hline & & SYG & sync_0565 & brown & 0.977 & -0.7 & $2.19 \mathrm{E}-05$ \\
\hline & & SYW & SYNW1957 & yellow & -0.964 & -0.3 & 0.0007 \\
\hline \multirow[t]{4}{*}{-} & \multirow[t]{4}{*}{ ABC transporter, ATP binding component } & PMM & PMM0290 & red & -0.813 & 0.4 & 0.002 \\
\hline & & PMT & PMT1635 & turquoise & -0.611 & -0.3 & 0.005 \\
\hline & & SYG & sync_2588 & darkturquoise & -0.746 & -0.2 & 0.0207 \\
\hline & & SYW & SYNW0320 & black & 0.791 & 0.7 & 0.015 \\
\hline \multirow[t]{4}{*}{ K02639 } & \multirow[t]{4}{*}{ ferredoxin } & PMM & PMM0898 & red & -0.813 & -0.1 & 0.035 \\
\hline & & PMT & PMT1429 & purple & 0.613 & 0.6 & 0.011 \\
\hline & & SYG & sync_1953 & turquoise & -0.806 & -0.6 & 0.006 \\
\hline & & SYW & SYNW1277 & midnightblue & -0.722 & 0.4 & 0.012 \\
\hline
\end{tabular}


tetrahydrobiopterin synthase (pts) (Table 3). However, not all of these genes shared the same expression patterns cross species, except for one gene, fts $W$, which was downregulated in all four species under both iron starvation and copper toxicity. Encoding a cell-division intergral membrane protein, fts $W$ has been found essential in E. coli [45] and was required for Z-ring stabilization during sporulation septation in Streptomyces coelicolor [46]. Both FtsQ (another cell division protein) and FtsW are indispensable to Synechocystis and their depletion led to slow growth and giant cells [47]. Recent study indicated that FtsW was also directly involved in DNA damage checkpoint coordinately interacting with an SOS regulon in Caulobacter crescentus [48], and the down-regulation of this gene might inhibit cell division [49]. Both iron depletion and high copper can cause oxidative stress and DNA damage [25,31], it could thus be speculated that $f t z W$ gene may play an important role in cellular defense to environmental disturbances.

Several other stress-regulated genes detected in all four selected cyanobacteria species also showed different direction of regulation (i.e. up- or down-regulation) in all species. For instance, ABC transporter ATP binding component [50] was up-regulated in PMM and SYW but down-regulated in PMT and SYG; carboxypeptidase Taq metallopeptidase was up-regulated in SYW but downregulated in the other 3 species. Isocitrate dehydrogenase $\left(\mathrm{NAD}^{+}\right)$(idh3), involved in several metabolism pathways like citrate cycle and secondary metabolite biosynthesis, was up-regulated in PMM but down-regulated in PMT, SYG and SYW. Similar to idh3, LysM domain gene encoding a LysM-containing protein involved in bacterial cell wall degradation [51], was also up-regulated in PMM but down-regulated in PMT, SYG and SYW. Interestingly, gene encoding PurH catalyzing the last two steps of de novo purine biosynthesis in bacteria and eukaryotes [52], was up-regulated in iron depletion but down-regulated in copper toxicity treatments.

Phycocyanobilin:ferredoxin oxidoreductase, PcyA, catalyzes biosynthesis of the phycobili-protein and phytochrome chromophore precursor phycocyanobilin [53]. Phycocyanobilin can serve as a light-harvesting pigment in the photosynthetic light-harvesting structures of cyanobacteria called phycobilisomes [54]. Its encoding gene was found up-regulated in PMT and SYW but down-regulated in PMM and SYG.

Ferredoxin coding gene was up-regulated in PMT and SYW but down-regulated in PMM and SYG. Level of cellular ferredoxin and flavodoxin was previously proposed as an indicator of iron stress in cyanobacteria [55]; however, a recent analysis about iron availability and production of ferredoxin in Antarctic sea ice diatoms showed no strong correlation between their levels and stress conditions [56]. Our results here supported the argument that ferredoxin level alone may not be a strong evidence of iron limited growth.

Another gene responsive in both iron and copper perturbations was pts encoding an enzyme involved in the early biosynthetic pathway of pteridines in cyanobacteria [57]. The gene was found up-regulated in species of PMT, SYW and SYG. The role of cyanopterin in $\mathrm{UV} /$ blue light single transduction of cyanobacterium Synechocystis sp. PCC 6803 phototaxis was verified recently [58]. Cyanobacteria are well known for producing high amounts of pteridine glycosides, and pterin compounds have been suggested as possible photoreceptor pigments in some stress responses that are induced by UV and blue light, probably due to their chemical and photophysical properties [59].

With the aid of WCGNA network analysis, it is also possible to obtain novel insights into molecular responses to environmental changes which will otherwise not be achieved by analyzing data from a single species. For example, a total of 27 shared metal-responsive KEGG pathways between two metal stresses were determined by the WGCNA analysis (Additional file 11: Table S7), which included 20 pathways of "amino acid, nitrogen, sulfur metabolism", 2 pathways of "photosynthesis and porphyrin and chlorophyll metabolism", 1 pathway each for "ABC transporters", "oxidative phosphorylation", "DNA mismatch repair", and "biosynthesis of secondary metabolites", respectively. Based on the fact that they responded to both iron depletion and high copper toxicity in multiple cyanobacteria species, we proposed that these pathways may represent the core metal-responsive pathways. Moreover, among all 40 signature genes of the PMT-greenyellow module, many were involved in amino acid metabolism, secondary metabolites, ABC transporters, and homologous recombination. PMM, originally isolated from surface water of the Mediterranean ocean, is high-light adapted with higher copper tolerance [25]. Probably due to these physiological differences, some highly correlated modules detected in PMT were not seen in the marine coastal species PMM, although a large number of active KEGG pathways detected were commonly shared by two species under the iron depletion condition (Table 2). It has been speculated that the better adaptation of PMT to lower iron concentration may be due to more efficient iron transport systems, better protection of iron stress and lower requirement for iron during growth [25].

\section{Divergent hub genes for different cyanobacteria species under metal stress}

Hub genes which have high connectivity within the networks are considered to play important roles in different phenotypes, such as yeast viability [60] and 
drought stress [61]. Recent work on cyanobacteria showed that there existed big divergence of gene expression patterns between cyanobacteria species from different ecological environments [25,31].

The genes might be considered as hub genes to a specific stress with the most connected genes in the responsive modules developed from the WGCNA analysis $[60,61]$. One of the major goals of this cross-species analysis was to discover the hub genes in each cyanobacteria species under specific environmental stimuli. The WGCNA analysis showed that some genes were connected with more than 8 other genes. Based on the annotated genes in the WGCNA modules, we obtained modest lists of orthologous hub genes for PMT responsive to iron depletion (number of the genes: 16) and SYW to copper toxicity (16), and shorter lists for PMM (10) and SYG (6). Surprisingly, no overlap of the hub genes was found between species, suggesting they may be unique to each species. For example, in PMT, the hub genes mainly encode metabolic enzymes, like GMP synthase (glutamine-hydrolysing), ketol-acid reductoisomerase; while in PMM, the genes were involved in photosynthesis system (psaI and $r b c L$ ) and two-component system $(p h o B)$. Nearly half of the hub genes in SYW encode ribosomal proteins (7) and oxidative phosphoraylation proteins (atpfla and atpf1d), compared to SYG hub genes with functions of metabolic enzymes and photosysm II PsbK protein and phycoerythrin-associated linker proteins (Additional file 12: Table S8). In terms of the functionally unknown genes in the modules (Additional file 13: Table S9), the analysis showed that they were also included in some gene clusters. For instance, PMT101-102-107 (black module), PMM1395-1396 (green module), sync_2434-2436 (purple module) were grouped together, while SYWN01930194 and SYWN0319-0320 were not in the same module. The function of these stress-responsive gene clusters with unknown functions may worth further investigation [60].

Hub genes obtained through the WGCNA analysis also showed distinct genes involved in iron stress in different Prochlorococcus species, which provided important clues for the differential fitness of these two species at low iron concentrations: $i$ ) the existence of a unique gene, lipopolysaccharide transport system ATP-binding protein (ABC-2.LPSE.A) and the activation of a urea transport system permease protein (urtB) may cause a more efficient iron transport features in PMT; ii) downregulated ribosome genes $(r p-S 15, r p-L 3)$ in PMT could save energy for cell survival under low iron

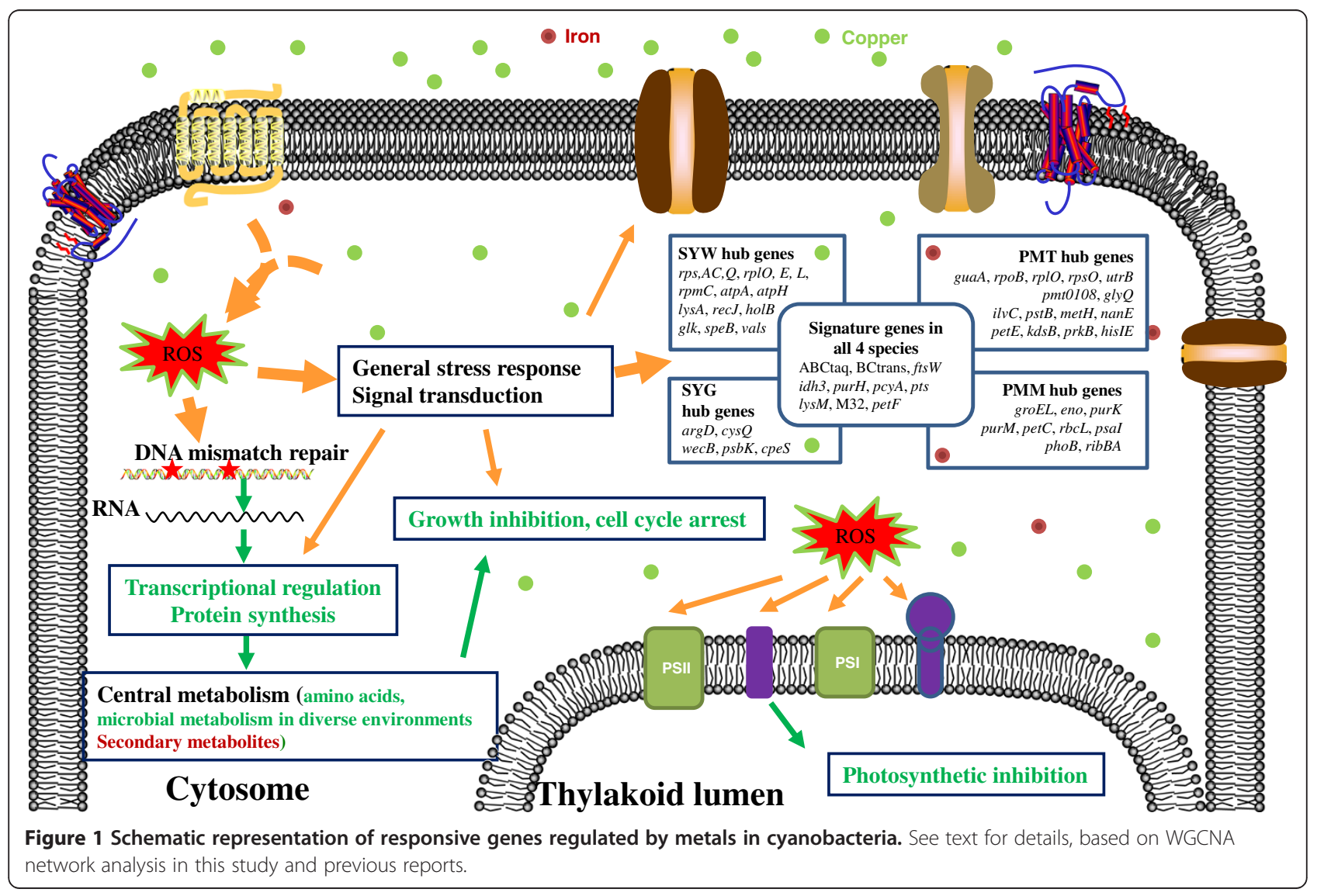


concentration; and iii) differentially expressed hypothetical genes with unknown functions in some of the highly correlated modules, especially those in PMT, may play important roles in the differential fitness [62]. Based on the combined analysis of both modules and hub genes, our study indicated that different Prochlorococcus species employed some specific metabolic pathways, together with general stress responses, to survive in different environments. Based our WGCNA analysis results and a previous review [63], the representation of responsive networks regulated by metals in cyanobacteria was schemed in Figure 1.

\section{Conclusions}

In the study, we have provided evidences that crossspecies WGCNA analysis could be a powerful tool to investigate the molecular mechanisms underlying the response of microbes to their environments. Based on the shared genes identified between all 4 cyanobacteria species evaluated in this study, we have found some similar strategies adopted by the cyanoabcteria to deal with different stresses. Further investigation of these shared genes showing differential expression in various species may shed light on their adaption in unique environment. In addition, our cross-species analysis has found some obvious differences between two metal treatments. The extraction of hub genes highly connected with other genes in cyanobacteria genomes may be valuable in determining essential genes for cyanobacteria [64]. Finally, we also noticed many hypothetical genes with function unknown were grouped with important genes in some stress-correlated modules. Although they were not discussed in details in this paper due to the fact of no much functional information is available for them, we believe that further deciphering their functions will provide new insights to the stress responses in cyanobacteria.

\section{Methods}

\section{Dataset acquisition}

Microarray datasets of four cyanobacteria species, Prochlorococcus MED4 (PMM), Prochlorococcus MIT9313 (PMT), Synechococcus 9311 (SYG) and Synechococcus WH 8102 (SYW) [25,31], were downloaded from the Gene Expression Omnibus (GEO) [65] (Table 1). The experiments were designed for with iron $(1 \mu \mathrm{M})$ as control and without iron as treatment for Prochlorococcus at different time $(0,12,24$ and $48 \mathrm{~h}$ for PMM and 0, 26, 28

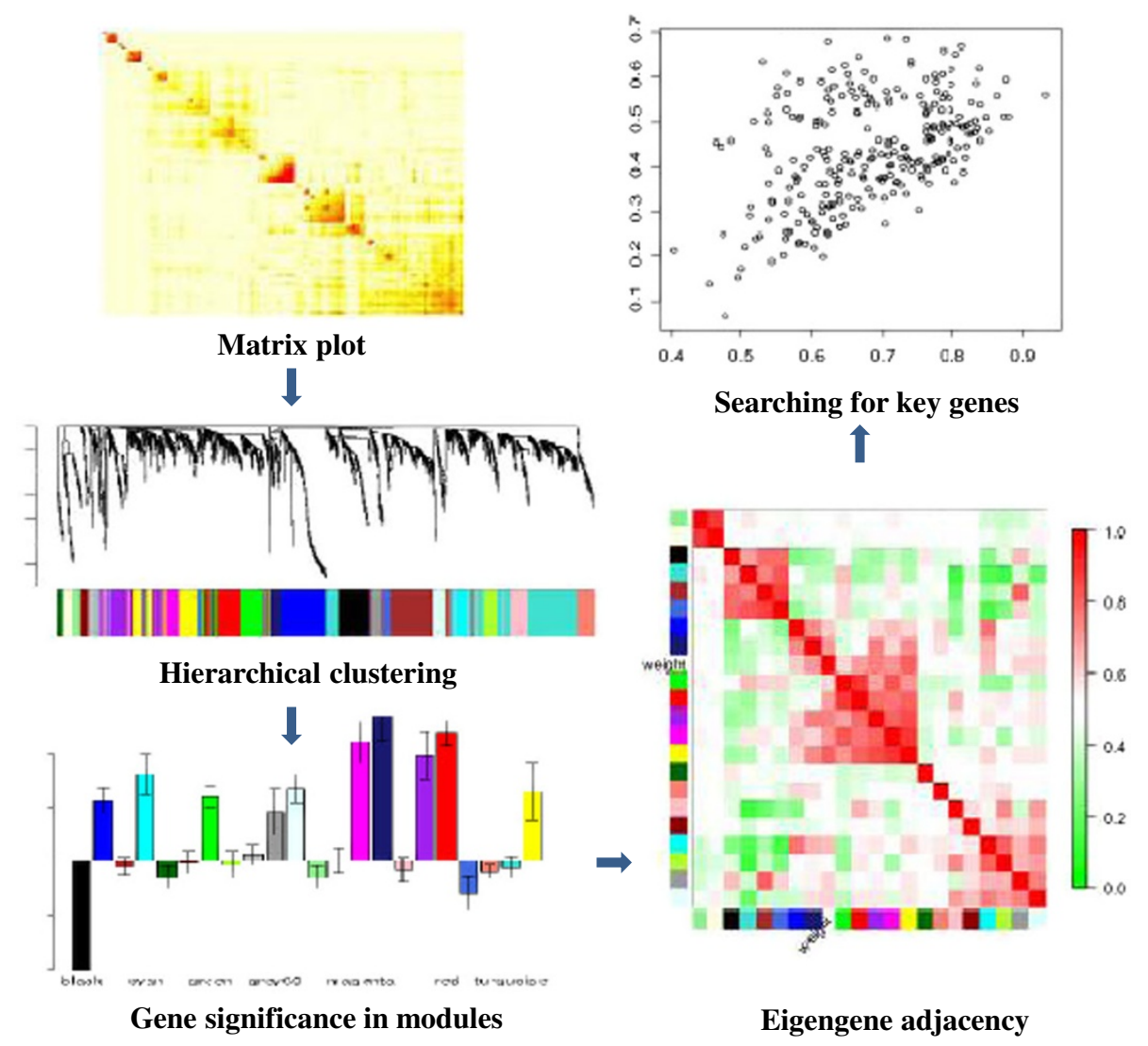

Figure 2 Scheme of the WGCNA work flow used in this study. Details of the WGCNA work flow referred in the text and reference (13). 
and $53 \mathrm{~h}$ for PMT based on their growth stages) and $2 \mathrm{~h}$ of copper treatments with concentrations of $1 \mu \mathrm{M}$ (SYW) and $0.1 \mu \mathrm{M}$ (SYG) [25,31]. The experimental details can be found in the original publication [25,31]. To compile an extensive set of comparable data, we collected all relevant datasets from the two experiments and then removed the outliers or samples forming an out-group to ensure the results reflecting the real biological response to different treatments. Specifically, we first performed a hierarchical clustering analysis (ward method) using (1-r) as distance for all samples, where $r$ is the pair-wise (between any two pairs of samples) correlation coefficient for the expression of all genes (probesets). Then we compared the tree structure with the status of treatment condition. For samples contradicting with the status of treatment, we excluded them from the downstream network analysis. For example, we found that in both Prochlorococcus datasets, all samples at 0 hour clustered together no matter they were depleted with iron or had iron, suggesting that their expression was irrelevant to iron status. Including them will compromise the power of the analysis. Therefore, we excluded all samples at 0 hour from our analysis. The updated genome annotation for all four species was downloaded from NCBI and the Comprehensive Microbial Resource (CMR) of TIGR (http://www.tigr.org/ CMR) [32-34]. KEGG Pathway and COGs (Cluster of Orthologous Groups of proteins) information was obtained from the KEGG Pathway Databases (http://www. genome.jp/kegg/pathway.html) and the COG Database of NCBI (http://www.ncbi.nlm.nih.gov/books/NBK21090/).

\section{Network construction}

For each cyanobacteria species, we created a transcriptional network from the microarray data, first by calculating weighted Pearson correlation matrices corresponding to gene expression, and then by following the standard procedure of WGCNA to create the networks [13]. Figure 2 schemes the methodology used in this study. Briefly, weighted correlation matrices were transformed into matrices of connection strengths using a power function [13]. These connection strengths were then used to calculate topological overlap (TO), a robust and biologically meaningful measurement that encapsulates the similarity of two genes' co-expression relationships with all other genes in the network [13]. Hierarchical clustering based on TO was used to group genes with highly similar co-expression relationships into modules. From the expression data we followed the protocols of WGCNA $[60,66]$ to create within-species consensus networks. Gene dendrograms were obtained by average linkage hierarchical clustering (Additional file 14: Figure S5, A-D), while the color row underneath the dendgram showed the module assignment determined by the
Dynamic Tree Cut (WGCNA). The network for each module was generated with the minimum spanning tree with dissimilarity matrix from WGCNA. To focus on biological interpretation, in this study, only genes with functional annotation were included. The modules with $r>0.6$, and $p$-value less than 0.05 were extracted for further investigation. KEGG pathway analysis was applied to detect the common pathways involved among different modules. Hub genes were screened by the links $(\geq 8)$ in the modules strongly associated with phenotype (iron depletion or copper toxicity, based on correlation coefficient $r>0.6$ ).

To establish cross-species signature genes in this analysis, and to define conservation in terms of stress response across cyanobacteria species, phenotype-correlated modules and the associated genes in each species were extracted. To assess co-expression perseveration between the species on a module-by-module basis, we first calculated a value of the module membership (MM) - a measure of how well each gene correlates with the first principal component of gene expression within a module, termed as the module eigengene. We then imposed a threshold based on MM values $(r, p)$ to make the final module assignments.

\section{Additional files}

Additional file 1: Figure S1. Clustering of the transcriptomic datasets under iron and copper treatments in different species. The grouped datasets with solid red color in "HighCopper" and "Iron" suggested high confident grouping of treated groups from controls.

Additional file 2: Figure S2. Association between phenotypes and identified transcriptional modules in different species. Each of the identified transcriptional modules was indicated by different colors, and their association with the phenotypes was indicated by $p$-values.

Additional file 3: Figure S3. Overlap of the detected responsive modules within and between cyanobacteria species. The lines connected different cyanobacteria species means there are some genes shared between specific modules.

Additional file 4: Table S1. genes and pathways in SYW-yellow module.

Additional file 5: Table S2. genes and pathways in SYW-Green yellow module.

Additional file 6: Table S3. genes and pathways in SYG-dark green module.

Additional file 7: Table S4. genes and pathways in SYG-brown module.

Additional file 8: Figure S4. Shared responsive genes in cyanobacteria species to iron depletion and copper toxicity treatments, respectively, and shared responsive genes between treatments in all cyanobacteria species. Nine shared genes among all 4 cyanobacteria species were listed in Table 3.

Additional file 9: Table S5. shared genes in iron treatments. The color of each gene indicates differential change: red is up-regulated and green is down-regulated.

Additional file 10: Table S6. shared signature genes in copper treatments. The color of each gene indicates the differential change, red is up-regulated and green is down-regulated.

Additional file 11: Table S7. Pathways shared in all 4 species. 
Additional file 12: Table S8. Hub genes with known functions in different cyanobacteria species. The same color from KO ID and the gene names indicate the hub genes in each species.

Additional file 13: Table S9. Hub genes with unknown functions in different cyanobacteria species.

Additional file 14: Figure S5. Hierarchical clustering tree using the topological overlap dissimilarity. Tree branches have been colored by module membership. A, B) PMT, PMM to iron, respectively; C, D) SYW, SYG to copper, respectively. Please refer the text for details of the analysis.

\section{Abbreviations}

ABC: ATP-binding cassette; FtsW: Cell division protein W; FtsQ: Cell division protein Q; HK: Histidine kinase; Idh3: Isocitrate dehydrogenase (nad ${ }^{+}$); kegg: Kyoto encyclopedia of genes and genomes; pcya: Phycocyanobilin: ferredoxin oxidoreductase; pts: 6-pyruvoyl tetrahydrobiopterin synthase: pmm: Prochlorococcus med4; pmt: Prochlorococcus mit9313; purh: Phosphoribosylaminoimidazolecarboxamide formyltransferase/imp cyclohydrolase; rr: Response regulator; syg: Synechococcus 9311; syw: Synechococcus wh 8102; tca: Citrate cycle; urtb: Urea transport system permease protein; WGCNA: Weighted Gene Co-expression Network Analysis.

\section{Competing interests}

The authors declare that they have no competing interests.

\section{Authors' contributions}

JW, GW, LC and WZ were responsible for the study design and coordination, contributed to the bioinformatics analysis and wrote the manuscript. JW and GW performed WGCNA and were responsible for the biological analysis. LC contributed to the data acquisition. All authors read and approved the final manuscript.

\section{Acknowledgements}

This study was funded by the National Basic Research Program of China (National "973" program) (No. 2011CBA00803 and No. 2012CB721101), and National High-tech R\&D Program (National "863" program) (No. 2012AA02A707). The authors would also like to thank Tianjin University and the "985 Project" of Ministry of Education for their financial supports in establishing the research laboratory.

\section{Author details}

${ }^{1}$ School of Chemical Engineering \& Technology, Tianjin University, Tianjin 300072, People's Republic of China. ${ }^{2}$ Key Laboratory of Systems Bioengineering, Ministry of Education, Tianjin 300072, People's Republic of China. ${ }^{3}$ Department of Biological Sciences, University of Maryland at Baltimore County, Baltimore, MD 21250, USA.

Received: 4 October 2012 Accepted: 13 February 2013 Published: 19 February 2013

\section{References}

1. Lu Y, Huggins P, Bar-Joseph Z: Cross species analysis of microarray expression data. Bioinformatics 2009, 25(12):1476-1483.

2. Altschul SF, Gish W, Miller W, Myers EW, Lipman DJ: Basic local alignment search tool. J Mol Biol 1990, 215(3):403-410.

3. Cai J, Xie D, Fan ZW, Chipperfield H, Marden J, Wong WH, Zhong S: Modeling co-expression across species for complex traits: insights to the difference of human and mouse embryonic stem cells. Plos Comput Biol 2010, 6:3.

4. Arbeitman MN, Furlong EEM, Imam F, Johnson E, Null BH, Baker BS, Krasnow MA, Scott MP, Davis RW, White KP: Gene expression during the life cycle of Drosophila melanogaster. Science 2002, 297(5590):2270-2275.

5. Nau GJ, Richmond JFL, Schlesinger A, Jennings EG, Lander ES, Young RA Human macrophage activation programs induced by bacterial pathogens. P Natl Acad Sci USA 2002, 99(3):1503-1508.

6. Correa A, Lewis AZ, Greene AV, March IJ, Gomer RH, Bell-Pedersen D: Multiple oscillators regulate circadian gene expression in Neurospora. P Natl Acad Sci USA 2003, 100(23):13597-13602.

7. Bergmann S, Ihmels J, Barkai N: Similarities and differences in genomewide expression data of six organisms. Plos Biol 2004, 2(1):85-93.
8. Sharan R, Suthram S, Kelley RM, Kuhn T, McCuine S, Uetz P, Sittler T, Karp $\mathrm{RM}$, Ideker T: Conserved patterns of protein interaction in multiple species. P Natl Acad Sci USA 2005, 102(6):1974-1979.

9. Odom DT, Dowell RD, Jacobsen ES, Gordon W, Danford TW, Maclsaac KD, Rolfe PA, Conboy CM, Gifford DK, Fraenkel E: Tissue-specific transcriptional regulation has diverged significantly between human and mouse. Nat Genet 2007, 39(6):730-732.

10. lori G, Precup OV: Weighted network analysis of high-frequency crosscorrelation measures. Phys Rev E 2007, 75(3):036110.

11. Langfelder $\mathrm{P}$, Horvath S: WGCNA: an R package for weighted correlation network analysis. BMC Bioinforma 2008, 9:559.

12. DiLeo MV, Strahan GD, den Bakker M, Hoekenga OA: Weighted Correlation Network Analysis (WGCNA) applied to the tomato fruit metabolome. PLoS One 2011, 6(10):e26683.

13. Zhang $B$, Horvath $S$ : A general framework for weighted gene coexpression network analysis. Stat App/ Genet Mo B 2005, 4:17.

14. Horvath S, Zhang B, Carlson M, Lu KV, Zhu S, Felciano RM, Laurance MF, Zhao W, Qi S, Chen Z, et al: Analysis of oncogenic signaling networks in glioblastoma identifies ASPM as a molecular target. P Natl Acad Sci USA 2006, 103(46):17402-17407

15. Aten JE, Fuller TF, Lusis AJ, Horvath S: Using genetic markers to orient the edges in quantitative trait networks: the NEO software. BMC Syst Biol 2008, 2:34

16. Oldham MC, Horvath S, Geschwind DH: Conservation and evolution of gene coexpression networks in human and chimpanzee brains. Proc Natl Acad Sci U S A, 103(47):17973-17978.

17. Park CC, Gale GD, de Jong S, Ghazalpour A, Bennett BJ, Farber CR, Langfelder P, Lin A, Khan AH, Eskin E, et al: Gene networks associated with conditional fear in mice identified using a systems genetics approach. BMC Syst Biol 2011, 5:43

18. Allen JD, Xie Y, Chen M, Girard L, Xiao G: Comparing statistical methods for constructing large scale gene networks. PLoS One 2012, 7(1):e29348.

19. Giordano $\mathrm{M}$, Beardall J, Raven $\mathrm{JA}: \mathrm{CO}_{2}$ concentrating mechanisms in algae: mechanisms, environmental modulation, and evolution. Annu Rev Plant Biol 2005, 56:99-131

20. Li WKW: Primary production of prochlorophytes, cyanobacteria, and eukaryotic ultraphytoplankton - measurements from flow cytometric sorting. Limnol Oceanogr 1994, 39(1):169-175.

21. Moore JK, Doney SC, Lindsay K: Upper ocean ecosystem dynamics and iron cycling in a global three-dimensional model. Global Biogeochem Cy 2004, 18(4):GB4028.

22. Boyd PW, Jickells T, Law CS, Blain S, Boyle EA, Buesseler KO, Coale KH, Cullen JJ, de Baar HJW, Follows M, et al: Mesoscale iron enrichment experiments 19932005: synthesis and future directions. Science 2007, 315(5812):612-617.

23. Mann EL, Ahlgren N, Moffett JW, Chisholm SW: Copper toxicity and cyanobacteria ecology in the Sargasso Sea. Limnol Oceanogr 2002, 47(4):976-988

24. Rusch DB, Martiny AC, Dupont $C L$, Halpern AL, Venter JC: Characterization of Prochlorococcus clades from iron-depleted oceanic regions. $P$ Natl Acad Sci USA 2010, 107(37):16184-16189.

25. Thompson AW, Huang K, Saito MA, Chisholm SW: Transcriptome response of high- and low-light-adapted Prochlorococcus strains to changing iron availability. ISME J 2011, 5(10):1580-1594.

26. Wilhelm SW, Trick CG: Physiological profiles of Synechococcus (Cyanophyceae) in Iron-limiting continuous cultures. J Phycol 1995, 31(1):79-85

27. Nodop A, Pietsch D, Hocker R, Becker A, Pistorius EK, Forchhammer K, Michel KP: Transcript profiling reveals new insights into the acclimation of the mesophilic fresh-water cyanobacterium Synechococcus elongatus PCC 7942 to iron starvation. Plant Physiol 2008, 147(2):747-763.

28. Liu SW, Qiu BS: Different responses of photosynthesis and flow cytometric signals to iron limitation and nitrogen source in coastal and oceanic Synechococcus strains (Cyanophyceae). Mar Biol 2012, 159(3):519-532.

29. Mann EL, Chisholm SW: Iron limits the cell division rate of Prochlorococcus in the eastern equatorial Pacific. Limnol Oceanogr 2000, 45(5):1067-1076.

30. Brand LE, Sunda WG, Guillard RRL: Reduction of marine-phytoplankton reproduction rates by copper and cadmium. J Exp Mar Biol Ecol 1986, 96(3):225-250

31. Stuart RK, Dupont CL, Johnson DA, Paulsen IT, Palenik B: Coastal strains of marine Synechococcus species exhibit Increased tolerance to copper 
shock and a distinctive transcriptional response relative to those of open-ocean strains. Appl Environ Microb 2009, 75(15):5047-5057.

32. Palenik B, Brahamsha B, Larimer FW, Land M, Hauser L, Chain P, Lamerdin J, Regala W, Allen EE, McCarren J, et al: The genome of a motile marine Synechococcus. Nature 2003, 424(6952):1037-1042.

33. Rocap G, Larimer FW, Lamerdin J, Malfatti S, Chain P, Ahlgren NA, Arellano A, Coleman M, Hauser L, Hess WR, et al: Genome divergence in two Prochlorococcus ecotypes reflects oceanic niche differentiation. Nature 2003, 424(6952):1042-1047.

34. Palenik B, Ren $\mathrm{QH}$, Dupont $\mathrm{CL}$, Myers GS, Heidelberg JF, Badger JH, Madupu R, Nelson WC, Brinkac LM, Dodson RJ, et al: Genome sequence of Synechococcus CC9311: insights into adaptation to a coastal environment. P Natl Acad Sci USA 2006, 103(36):13555-13559.

35. Zhu YH, Graham JE, Ludwig M, Xiong W, Alvey RM, Shen GZ, Bryant DA: Roles of xanthophyll carotenoids in protection against photoinhibition and oxidative stress in the cyanobacterium Synechococcus sp strain PCC 7002. Arch Biochem Biophys 2010, 504(1):86-99.

36. Ledala N, Sengupta M, Muthaiyan A, Wilkinson BJ, Jayaswal RK: Transcriptomic response of Listeria monocytogenes to iron limitation and fur Mutation. Appl Environ Microb 2010, 76(2):406-416.

37. Ihrig J, Hausmann A, Hain A, Richter N, Hamza I, Lill R, Muhlenhoff U: Iron Regulation through the back door: iron-dependent metabolite levels contribute to transcriptional adaptation to iron deprivation in Saccharomyces cerevisiae. Eukaryot Cell 2010, 9(3):460-471.

38. Serkin $C D$, Seifert HS: Iron availability regulates DNA recombination in Neisseria gonorrhoeae. Mol Microbiol 2000, 37(5):1075-1086.

39. Lovcinsky M, Dedic R, Psencik J, Benesova J, Stys D, Hala J: Spectroscopic characterization of pigment binding proteins in normal-grown and ironstressed thermophilic cyanobacteria Synechococcus sp. J Mol Struct 1999 481:577-580.

40. Guikema JA, Sherman LA: Organization and function of chlorophyll in membranes of cyanobacteria during iron starvation. Plant Physiol 1983, 73(2):250-256

41. Boekema EJ, Hifney A, Yakushevska AE, Piotrowski M, Keegstra W, Berry S, Michel KP, Pistorius EK, Kruip J: A giant chlorophyll-protein complex induced by iron deficiency in cyanobacteria. Nature 2001, 412(6848):745-748.

42. Shavyrina OB, Gapochka LD, Azovskii Al: Development of tolerance for copper in cyanobacteria repeatedly exposed to its toxic effect. Biol Bull 2001, 28(2):183-187.

43. Michel KP, Pistorius EK: Adaptation of the photosynthetic electron transport chain in cyanobacteria to iron deficiency: the function of IdiA and IsiA. Physiol Plantarum 2004, 120(1):36-50.

44. Garcia-Villada L, Rico M, Altamirano MM, Sanchez-Martin L, Lopez-Rodas V, Costas E: Occurrence of copper resistant mutants in the toxic cyanobacteria Microcystis aeruginosa: characterisation and future implications in the use of copper sulphate as algaecide. Water Res 2004, 38(8):2207-2213.

45. Boyle DS, Khattar MM, Addinall SG, Lutkenhaus J, Donachie WD: ftsW is an essential cell-division gene in Escherichia coli. Mol Microbiol 1997, 24(6):1263-1273.

46. Mistry BV, Del Sol R, Wright C, Findlay K, Dyson P: FtsW is a dispensable cell division protein required for Z-ring stabilization during sporulation septation in Streptomyces coelicolor. J Bacteriol 2008, 190(16):5555-5566.

47. Marbouty M, Mazouni K, Saguez C, Cassier-Chauvat C, Chauvat F: Characterization of the Synechocystis strain PCC 6803 penicillin-binding proteins and cytokinetic proteins FtsQ and FtsW and their network of interactions with ZipN. J Bacterio/ 2009, 191(16):5123-5133.

48. Fraipont $C$, Alexeeva $S$, Wolf $B$, van der Ploeg $R$, Schloesser $M$, den Blaauwen T, Nguyen-Disteche M: The integral membrane FtsW protein and peptidoglycan synthase PBP3 form a subcomplex in Escherichia coli. Microbiol-Sgm 2011, 157:251-259

49. Modell JW, Hopkins AC, Laub MT: A DNA damage checkpoint in Caulobacter crescentus inhibits cell division through a direct interaction with FtsW. Gene Dev 2011, 25(15):1662-1662.

50. Bliss JM, Garon CF, Silver RP: Polysialic acid export in Escherichia coli K1: the role of KpsT, the ATP-binding component of an ABC transporter, in chain translocation. Glycobiology 1996, 6(4):445-452.

51. Zhang XC, Cannon SB, Stacey G: Evolutionary genomics of LysM genes in land plants. BMC Evol Biol 2009, 9:183
52. Qiu XT, Yuan Y, Gao YX: Expression, purification, crystallization and preliminary X-ray diffraction crystallographic study of PurH from Escherichia coli. Acta Crystallogr F 2011, 67:1590-1594.

53. Frankenberg $N$, Lagarias JC: Phycocyanobilin: Ferredoxin oxidoreductase of Anabaena sp PCC 7120 - Biochemical and spectroscopic characterization. J Bio/ Chem 2003, 278(11):9219-9226.

54. Dammeyer T, Frankenberg-Dinkel N: Insights into phycoerythrobilin biosynthesis point toward metabolic channeling. J Biol Chem 2006, 281(37):27081-27089.

55. Fish W, Dalrymple M, Sandersloehr J: Cellular ferredoxin and flavodoxin levels as indicators of iron-stress in cyanobacteria. Abstr Pap Am Chem S 1987, 194:230. ENVR

56. Pankowski A, McMinn A: Iron availability regulates growth, photosynthesis, and production of ferredoxin and flavodoxin in Antarctic sea ice diatoms. Aquat Biol 2009, 4(3):273-288.

57. Lee SW, Lee HW, Chung HJ, Kim YA, Kim YJ, Hahn Y, Chung JH, Park YS: Identification of the genes encoding enzymes involved in the early biosynthetic pathway of pteridines in Synechocystis sp. PCC 6803. FEMS Microbiol Lett 1999, 179(1):181-181.

58. Moon YJ, Lee EM, Park YM, Park YS, Chung WI, Chung YH: The Role of Cyanopterin in UV/Blue light signal transduction of cyanobacterium Synechocystis sp PCC 6803 Phototaxis. Plant Cell Physiol 2010, 51(6):969-980.

59. Chung HJ, Kim YA, Kim YJ, Choi YK, Hwang YK, Park YS: Purification and characterization of UDP-glucose: tetrahydrobiopterin glucosyltransferase from Synechococcus sp PCC 7942. BBA-Gen Subjects 2000, 1524(2-3):183-188

60. Carlson MRJ, Zhang B, Fang ZX, Mischel PS, Horvath S, Nelson SF: Gene connectivity, function, and sequence conservation: predictions from modular yeast co-expression networks. BMC Genomics 2006, 7:40.

61. Zhang LD, Yu SW, Zuo KJ, Luo LJ, Tang KX: Identification of gene modules associated with drought response in rice by network-based Analysis. PLoS One 2012, 7(5):e33748,

62. Qiao J, Shao M, Chen L, Wang J, Wu G, Tian X, Liu J, Huang S, Zhang W: Systematic characterization of hypothetical proteins in Synechocystis sp. PCC 6803 reveals proteins functionally relevant to stress responses. Gene 2013, 512(1):6-15.

63. Castielli O, De la Cerda B, Navarro JA, Hervas M, De la Rosa MA: Proteomic analyses of the response of cyanobacteria to different stress conditions. FEBS Lett 2009, 583(11):1753-1758.

64. Helman Y, Tchernov D, Reinhold L, Shibata M, Ogawa T, Schwarz R, Ohad I, Kaplan A: Genes encoding A-type flavoproteins are essential for photoreduction of $\mathrm{O}_{2}$ in cyanobacteria. Curr Biol 2003, 13(3):230-235.

65. Edgar R, Domrachev M, Lash AE: Gene Expression Omnibus: NCBI gene expression and hybridization array data repository. Nucleic Acids Res 2002, 30(1):207-210

66. Ravasz E, Somera AL, Mongru DA, Oltvai ZN, Barabasi AL: Hierarchical organization of modularity in metabolic networks. Science 2002, 297(5586):1551-1555.

doi:10.1186/1471-2164-14-112

Cite this article as: Wang et al:: Cross-species transcriptional network analysis reveals conservation and variation in response to metal stress in cyanobacteria. BMC Genomics 2013 14:112.

\section{Submit your next manuscript to BioMed Central and take full advantage of:}

- Convenient online submission

- Thorough peer review

- No space constraints or color figure charges

- Immediate publication on acceptance

- Inclusion in PubMed, CAS, Scopus and Google Scholar

- Research which is freely available for redistribution 